\title{
Corticospinal excitability in idiopathic normal pressure hydrocephalus: a transcranial magnetic stimulation study
}

Jani Sirkka ${ }^{1 *} \mathbb{D}$, Laura Säisänen ${ }^{2,3}$, Petro Julkunen ${ }^{2,3}$, Mervi Könönen ${ }^{2,4}$, Elisa Kallioniemi ${ }^{2,5}$, Ville Leinonen ${ }^{1,6}$ and Nils Danner ${ }^{1}$

\begin{abstract}
Background: Idiopathic normal pressure hydrocephalus (iNPH) is a neurodegenerative disease with an unknown etiology. Disturbed corticospinal inhibition of the motor cortex has been reported in iNPH and can be evaluated in a noninvasive and painless manner using navigated transcranial magnetic stimulation (nTMS). This is the first study to characterize the immediate impact of cerebrospinal fluid (CSF) drainage on corticospinal excitability.

Methods: Twenty patients with possible or probable iNPH (16 women and 4 men, mean age 74.4 years, range 67-84 years), presenting the classical symptom triad and radiological findings, were evaluated with motor function tests (10-m walk test, Grooved Pegboard and Box \& Block test) and nTMS (silent period, SP, resting motor threshold, RMT and input-output curve, IO-curve). Evaluations were performed at baseline and repeated immediately after CSF drainage via lumbar puncture.

Results: At baseline, iNPH patients presented shorter SPs $(p<0.001)$ and lower RMTs $(p<0.001)$ as compared to normative values. Positive correlation was detected between SP duration and Box \& Block test (rho $=0.64, p=0.002$ ) in iNPH patients. CSF drainage led to an enhancement in gait velocity $(p=0.002)$ and a steeper IO-curve slope $(p=0.049)$.

Conclusions: Shorter SPs and lower RMTs in iNPH suggest impaired corticospinal inhibition and corticospinal hyperexcitability. The steeper IO-slope in patients who improve their gait velocity after CSF drainage may indicate a higher recovery potential. Corticospinal excitability correlated with the motor function of the upper limbs implying that the disturbance in motor performance in iNPH extends beyond the classically reported gait impairment.
\end{abstract}

Keywords: Idiopathic normal pressure hydrocephalus, Navigated transcranial magnetic stimulation, Lumbar puncture, Corticospinal excitability, Inhibition

\section{Background}

Idiopathic normal pressure hydrocephalus (iNPH) is a neurodegenerative disease with prevalence increasing with age $[1,2]$. Conventionally, the symptoms of iNPH have been characterized with the Hakim's triad which includes cognitive decline, urinary incontinence

\footnotetext{
*Correspondence: Janisir@uef.fi

${ }^{1}$ Neurocenter, Neurosurgery, Kuopio University Hospital and University of Eastern Finland, Kuopio, Finland
}

Full list of author information is available at the end of the article and gait disturbance [1]. Brain imaging typically shows enlargement of the cerebral ventricles and obliteration of parasagittal sulci [3]. The pathophysiology behind the symptoms has been attributed to abnormal dynamics of cerebrospinal fluid (CSF). Currently, the only effective treatment is CSF diversion by shunt surgery. However, for unclear reasons, shunt surgery does not offer alleviation of the symptoms in all patients. Depending on patient selection, less than $2 / 3$ improve their functional status while some patients experience only a subjective 
benefit. This emphasizes the need to identify biomarkers for shunt surgery responders in iNPH $[4,5]$.

While the gait dysfunction is the most prominent as well as the most treatment responsive symptom of iNPH, other disease-related motor dysfunctions have not been investigated as thoroughly. Recent studies have suggested that impaired balance may contribute to the gait dysfunction in iNPH [6, 7]. Furthermore, it has also been suggested that iNPH might affect upper limb function presenting as hypokinesia, which shares several features with the motor dysfunctions of Parkinson's disease [8]. However, the precise neuronal mechanisms that underlie the symptoms of iNPH remain unknown. Previous studies have shown that the corticospinal tract functions normally but the functional connectivity of central movement-regulating mechanisms may be disturbed [9-11].

In this study, we aimed to characterize the excitability of the motor cortex and the corticospinal tract in iNPH. For this purpose, transcranial magnetic stimulation (TMS) offers a non-invasive and painless research tool $[12,13]$ and until now, only three TMS studies have been carried out in iNPH $[10,11,14]$. The results have pointed towards abnormal corticospinal inhibition. However, in the previous studies, TMS was performed without navigation, for which reason the results could be postulated to derive from disease-related anatomical abnormalities. In iNPH patients ventriculomegaly and obliteration of cortical sulci may influence the effect of TMS, which is determined by the distance from the stimulation coil to the cortex $[3,15]$. In the current study, we evaluated the corticospinal excitability using a navigated TMS (nTMS) system, which enables magnetic resonance image (MRI) guided targeting and increases the spatial accuracy of the stimulation [15]. Furthermore, longitudinal examinations within subjects retain their comparability in nTMS [16].

The specific aims of the current study were (1) to characterize the disease related changes of corticospinal excitability in iNPH, (2) to evaluate associations between corticospinal excitability and clinical symptoms of iNPH, and (3) to assess the immediate impact of CSF drainage on corticospinal excitability in both upper and lower limbs using nTMS.

\section{Methods}

\section{Subjects}

Twenty patients were recruited for this study from an ongoing prospective iNPH study at Kuopio University Hospital (KUH) (Table 1). All patients had previously undergone a neurological evaluation and were referred to KUH for surgical evaluation due to suspected iNPH. The patients had probable or possible iNPH determined by the classical symptom triad [1] and brain imaging [3]. Other neurological comorbidities were

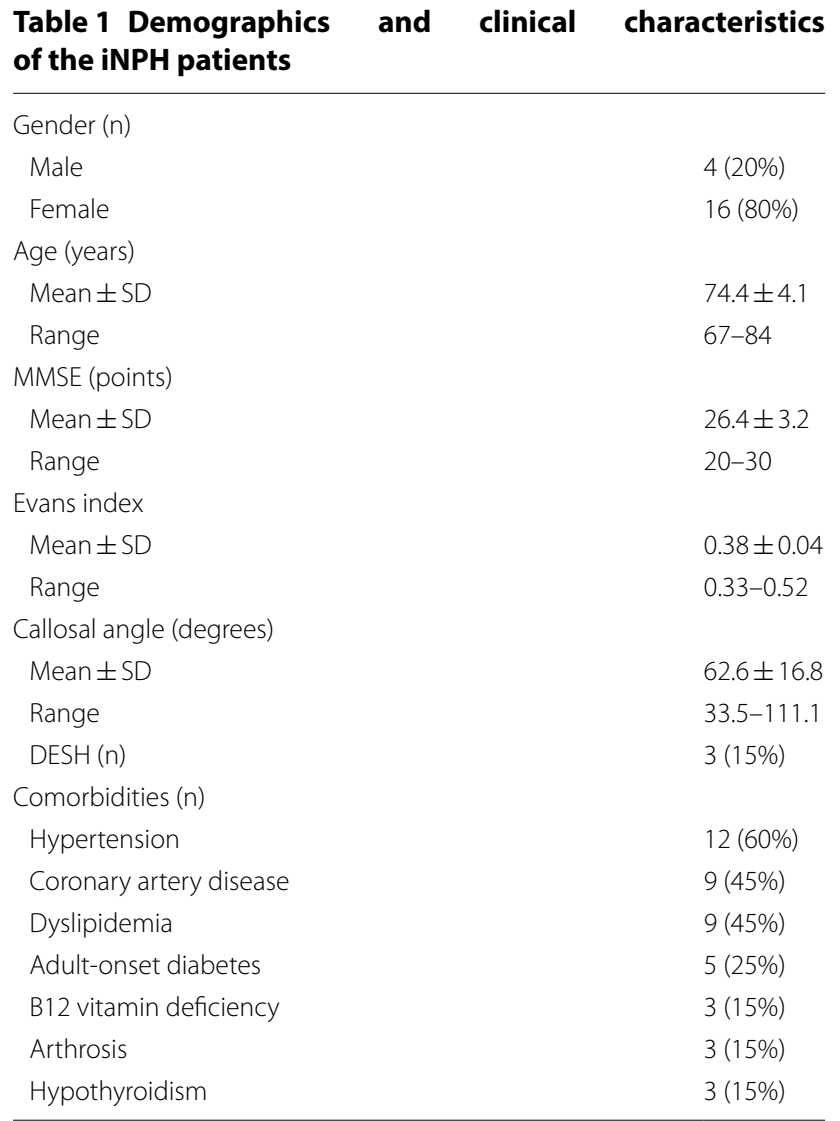

DESH disproportionately enlarged subarachnoid space [22], MMS mini mental state examination

regarded as exclusion criteria [3]. The patients were required not to use any medications with effects on TMS responses such as antidepressants or antipsychotics [17]. A score of at least 20 points in the Mini-Mental State Examination (MMSE) [18] was required to ensure fluent cooperation during the study and maintenance of attention throughout the TMS session, since TMS responses may be affected by the level of attention [19]. All patients were checked to be eligible for both TMS [20] and MRI [21]. The study protocol was approved by the local research ethics committee $(274 / 2016)$. All participants gave written informed consent. The patients did not receive any financial compensation for their participation.

A control population for the TMS responses was gathered from a pre-existing TMS database of KUH from two previous studies with healthy subjects $[23,24]$ in which nTMS was performed using an NBS version 2.2.0 (Nexstim Plc, Helsinki, Finland) system. In total 20 cases were selected based on age and gender (10 women and 10 men, mean age 69.2 years \pm 7.1 years, range 60-81). 


\section{Flow of the study}

The flow of the study is presented in Fig. 1. Each patient underwent the clinical assessments as well as nTMS and MRI studies twice, 1 day prior to the lumbar puncture (LP) and on the day of the LP. The clinical assessments were repeated immediately after the LP and were followed by nTMS and MRI within 4 to $6 \mathrm{~h}$.

\section{Clinical assessment}

At baseline, patients were evaluated using three domains of the iNPH scale: gait, neuropsychology and balance [25]. Gait was assessed in accordance with the iNPH scale with the total score of gait (TSG) in a 10-m walking test including ordinal rating, gait velocity and number of steps [25]. Balance was scored in accordance with the scale [25]. We applied a modified neuropsychology domain consisting of two tests, the Grooved Pegboard Test (GPT) (Lafayette Instruments, Lafayette, IN) and CERAD [26]. The GPT was used to evaluate fine motor function of upper limbs and CERAD to evaluate overall level of cognitive functions. In addition to the modified iNPH scale, the Box and Block Test (BBT) [27] was used to evaluate gross motor function of the upper limbs. In the TAP test (TT) $[28,29]$, CSF was drained up to $40 \mathrm{ml}$

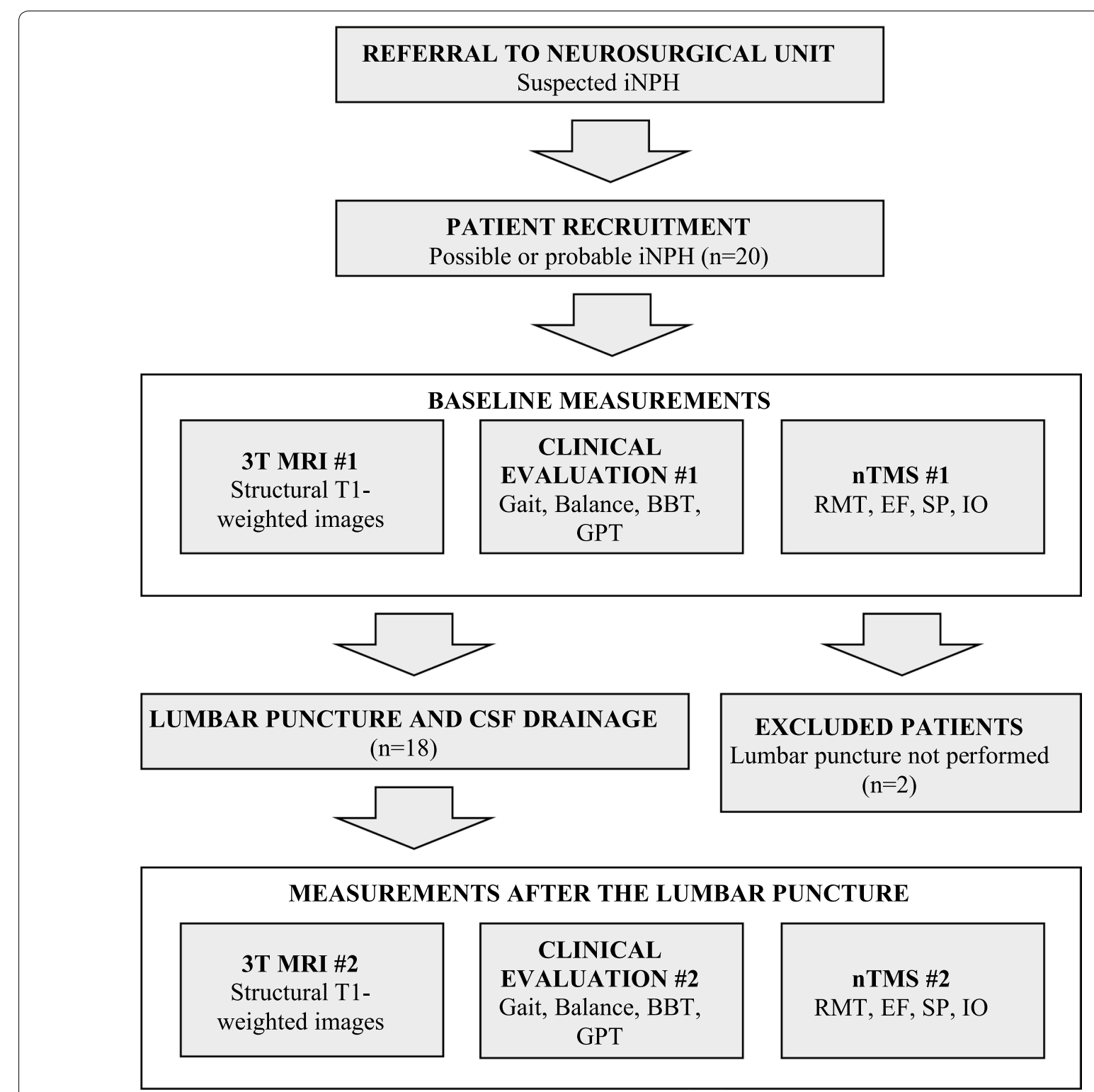

Fig. 1 Flow chart of the study. 3T MRI, 3 Tesla magnetic resonance imaging; SP silent period, RMT resting motor threshold, EF electric field, IO inputoutput curve, BBT Box and Block Test, GPT Grooved Pegboard Test, CSF cerebrospinal fluid 
via LP and all tests, with the exception of CERAD, were repeated identically as before the drainage (Fig. 1).

\section{Radiological imaging}

All subjects underwent clinical diagnostic MRI with a 3T MRI scanner (Philips Achieva, The Netherlands). The images were evaluated by an experienced neuroradiologist to exclude subjects with other neurological disorders than iNPH. Structural 3D T1-weighted images $(1 \mathrm{~mm} \times 1 \mathrm{~mm} \times 1 \mathrm{~mm}$ voxel size) were used for nTMS. Evans index [30] and callosal angle [31] were manually determined from the MR-images (Table 1).

\section{Navigated transcranial magnetic stimulation}

NTMS was performed using an NBS system (version 4.3.1, Nexstim Plc, Helsinki, Finland) and a figure-ofeight coil with biphasic pulse waveform. The nTMS protocol was performed identically before and after the LP. The primary motor cortex (M1) of the left hemisphere was stimulated and continuous electromyography (EMG) was recorded to register TMS-induced motor evoked potentials (MEPs) from the contralateral limb muscles with an integrated EMG system. When stimulating the M1 at the representation area of the upper limb, responses were registered from the first dorsal interosseous (FDI), the abductor pollicis brevis and the extensor carpi radialis muscles. When stimulating the cortical representation area of the lower limb, responses were registered from the abductor hallucis brevis (AHB), the tibialis anterior and the vastus lateralis muscles. MEPs from FDI to AHB were analyzed and other muscles were used as controls to detect and avoid concurrent muscle activation. A peak-to-peak amplitude of $50 \mu \mathrm{V}$ was the approval limit of MEPs. All responses with EMG-activity (above $50 \mu \mathrm{V}$ peak-to-peak amplitude) $50 \mathrm{~ms}$ before the stimulus were excluded from the analysis [32].

The nTMS protocol abided with the IFCN guidelines [33]. First, M1 was mapped in the vicinity of the hand knob to determine the hotspot (stimulation target) inducing MEPs with highest amplitude in the FDI muscle. At the stimulation target, resting motor threshold (RMT), silent period (SP) and input-output curve (IO) were measured (Table 2). Corticospinal excitability was evaluated as stimulator output percentage (\% of maximum stimulator output, \%-MSO) and with the calculated electric field (EF) strength $(\mathrm{V} / \mathrm{m})$. The RMT was measured at the stimulation target using an integrated iterative threshold assessment tool $[34,35]$. When the RMT is measured as \%-MSO it is influenced by the coil-to-cortex distance and may therefore theoretically be influenced by the CSF drainage. Therefore we used the calculated $\mathrm{EF}$ at the stimulation target as an additional measure of corticospinal excitability in order to eliminate the effect of possible anatomical alterations [16, 24, 36, 37]. The SP is a stimulation induced rest in voluntary muscle activation and its duration is considered to reflect the level of corticospinal inhibition [38]. The SP was calculated as an average duration of five trials induced by stimulating at $120 \%$ of the RMT [39]. The IO measurement was performed using a sequence of 160 stimulations with varying stimulation intensities $(80-150 \%$ of the RMT in $10 \%$ steps) and intervals (3-5 s) in a randomized order. A Boltzmann sigmoidal function was used to determine the maximum value (IO-max), the mid-point of the curve (IO-50) and the slope of the curve (IO-slope) [40]. After completing all measurements in the upper limb, the M1 was mapped for the representation area of the contralateral lower limb to determine the hotspot of the AHB. The

Table 2 Transcranial magnetic stimulation (TMS) responses measured in this study

\begin{tabular}{|c|c|c|c|c|}
\hline & Definition & Neuronal background & Interpretation & References \\
\hline Motor threshold & - & Glutamatergic system & $\begin{array}{l}\text { High motor threshold means } \\
\text { decreased corticospinal excit- } \\
\text { ability }\end{array}$ & {$[33,41,42]$} \\
\hline Resting motor threshold (RMT) & $\begin{array}{l}\text { Corticospinal excitability (\% of } \\
\text { maximum stimulator output) }\end{array}$ & - & - & - \\
\hline Electric field (EF) & $\begin{array}{l}\text { Corticospinal excitability } \\
\text { (induced electric field, V/m, at } \\
\text { the cortex) }\end{array}$ & - & - & - \\
\hline Silent period (SP) & Corticospinal inhibition & GABAergic system & $\begin{array}{l}\text { Long silent period means } \\
\text { increased corticospinal inhibi- } \\
\text { tion }\end{array}$ & {$[33,38,43]$} \\
\hline Input-output curve (IO-slope) & $\begin{array}{l}\text { Dose-dependent response to } \\
\text { TMS }\end{array}$ & $\begin{array}{l}\text { Synaptic connectivity and } \\
\text { plasticity }\end{array}$ & $\begin{array}{l}\text { The steepness of the IO-slope } \\
\text { reflects the level of synaptic } \\
\text { connectivity and plasticity }\end{array}$ & {$[40,44-46]$} \\
\hline
\end{tabular}


RMT and EF were determined in a similar manner as for the FDI in the upper limb.

\section{Statistical analysis}

The normality of distributions of each parameter was assessed with the Kolmogorov-Smirnov and Shapiro-Wilk tests. Non-parametric tests were used for all measures, since the majority were not normally distributed. Spearman's test was used to determine correlations between different measures in the iNPH patients. The iNPH group was divided into two subgroups based on the TT response: TT + : at least $10 \%$ improvement in gait velocity in the TAP test and TT-: under $10 \%$ improvement in gait velocity in the TAP test. TMS responses were compared between the iNPH and the control group (RMT/EF and SP) as well as between the divided iNPH subgroups using the independent-samples Mann-Whitney $U$ test. Measures with repeated measurements before and after the LP were statistically compared in pre- and post-LP situations with the Wilcoxon signed-rank test. P-values $<0.05$ were considered as statistically significant. Statistical analyses were performed with SPSS (version 24.0; IBM Corporation, Somers, NY).

\section{Results}

Twenty patients were evaluated for the study, two of which were excluded (LP could not be performed, $n=1$, and the symptoms were regarded as too mild to pursue with invasive testing, $\mathrm{n}=1$ ) after the baseline measurement (Fig. 1). At baseline (Table 3), the RMTs of upper and lower limbs were significantly lower in the iNPH patients as compared with the control group (upper limb: $\mathrm{U}=11.5$, $\mathrm{p}<0.001$; lower limb: $U=47.0, \mathrm{p}<0.001)$. Similarly, the cortical EF strength for the upper limbs was higher in the iNPH group ( $\mathrm{U}=79.5, \mathrm{p}=0.001)$. The iNPH group had also a significantly shorter SP duration than the control group $(\mathrm{U}=73.0, \mathrm{p}<0.001)$. In the $\mathrm{iNPH}$ group the RMTs of the upper and lower limbs correlated positively with each other $(\mathrm{rho}=0.56, \mathrm{p}=0.010)$ and the SP duration correlated positively with the BBT (rho $=0.64$, $\mathrm{p}=0.002$, Fig. 2). There were no other correlations between TMS responses and motor parameters (Table 4).

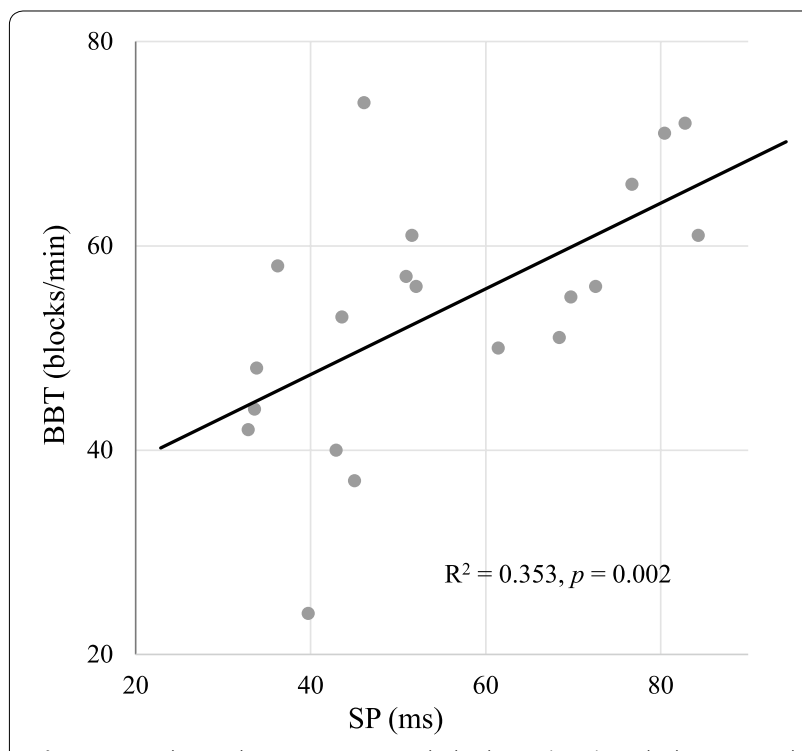

Fig. 2 Correlation between Box and Block test(BBT) and silent period (SP) duration at baseline

As a result of the LP and CSF drainage the iNPH patients showed a significant increase in TSG and gait velocity likewise increased step length reflected as a decrease in the number of steps. From TMS responses the IO-slope exhibited a significant increase after the LP (Table 5). The study population was divided into two nine-person subgroups based on the TT response with a predefined cut point of $10 \%$ improvement in gait velocity (TT+ and $\mathrm{TT}-$ ). Gait velocity was significantly slower in the TT+ subgroup as compared to the TTsubgroup in the pre-LP situation $(p=0.040)$. After the LP there were no significant differences in the motor parameters between the subgroups. The upper limb EF was statistically lower in the TT+ subgroup than in the $\mathrm{TT}-$ subgroup in the post-LP situation $(\mathrm{p}=0.024)$ but no significant within-subgroup changes were detected in the EF (Table 6). The IO-slope exhibited a significant increase after the LP in the TT+ subgroup $(\mathrm{p}=0.028$, Fig. 3), but not in the TT - subgroup (p>0.999).

Table 3 Silent period durations (SPs) and resting motor thresholds (RMTs) in iNPH patients and healthy controls

\begin{tabular}{lll}
\hline & iNPH $(\mathbf{n}=\mathbf{2 0})$ mean \pm SD, range & Control $(\mathbf{n}=\mathbf{2 0})$ mean \pm SD, range \\
\hline Upper limb SP (ms) & $55.3 \pm 17.7,32.9-84.3$ & $78.7 \pm 16.1,58.3-113.0$ \\
Upper limb RMT (\%-MSO) & $26.0 \pm 4.4,18.0-36.0$ & $45.4 \pm 12.2,29.0-71.0$ \\
(V/m) & $81.1 \pm 13.0,61.0-103.0$ & $103.6 \pm 23.6,62.0-152.0$ \\
Lower limb RMT (\%-MSO) & $41.5 \pm 7.6,27.0-60.0$ & $59.2 \pm 13.9,37.0-84.0$ \\
\hline
\end{tabular}

Significant differences are reported according to the Mann-Whitney $U$ test for independent samples

MSO maximum stimulator output 
Table 4 Baseline correlations between TMS responses, neuropsychologic performance and motor parameters in iNPH patients

\begin{tabular}{lcccrrr}
\hline & TSG & Gait velocity & Steps & BBT & GPT $^{\mathbf{a}}$ & CERAD \\
\hline Upper limb SP & 0.327 & 0.331 & -0.305 & $0.639^{*}$ & -0.311 & 0.095 \\
Upper limb RMT & -0.021 & -0.070 & 0.147 & 0.298 & -0.389 & -0.212 \\
Upper limb EF & -0.130 & -0.170 & 0.065 & 0.064 & -0.266 & -0.176 \\
Lower limb RMT & -0.043 & -0.098 & 0.058 & 0.089 & -0.185 & -0.147 \\
Lower limb EF & -0.208 & -0.139 & 0.084 & -0.007 & 0.058 & -0.059 \\
\hline
\end{tabular}

$\mathrm{n}=20$

SP silent period, RMT resting motor threshold, EF electric field, TSG total score of gait, BBT Box and Block Test, GPT Grooved Pegboard Test

*Indicatessignificant correlation ( $p=0.002$, Spearman's rho)

a One outlier excluded from statistical analyzes

Table 5 TMS responses and motor parameters before and after lumbar puncture

\begin{tabular}{lccc}
\hline & Pre-LP mean \pm SD & Post-LP mean \pm SD & $p$-value \\
\hline Upper limb SP (ms) & $55.5 \pm 17.4$ & $54.1 \pm 20.1$ & 0.227 \\
Upper limb RMT (\%-MSO) & $26.4 \pm 4.3$ & $26.1 \pm 3.9$ & 0.462 \\
Upper limb EF (V/m) & $82.1 \pm 13.0$ & $83.9 \pm 16.0$ & 0.601 \\
Lower limb RMT (\%-MSO) & $42.2 \pm 7.6$ & $43.1 \pm 8.3$ & 0.146 \\
Lower limb EF (V/m) & $149.3 \pm 44.2$ & $139.2 \pm 34.8$ & 0.586 \\
IO-max (mV) & $4.8 \pm 2.7$ & $4.9 \pm 2.8$ & 0.959 \\
IO-50 (\%) & $35.8 \pm 5.7$ & $36.4 \pm 4.9$ & 0.642 \\
IO-slope & $2.6 \pm 1.2$ & $3.1 \pm 1.4$ & $0.049^{*}$ \\
TSG (score) & $53.7 \pm 25.0$ & $58.0 \pm 25.2$ & $0.039^{*}$ \\
Gait velocity (m/s) & $0.86 \pm 0.27$ & $0.95 \pm 0.25$ & $0.002^{*}$ \\
Steps (number) & $23.1 \pm 6.7$ & $21.7 \pm 4.9$ & $0.017^{*}$ \\
BBT (pcs) & $54.5 \pm 12.5$ & $54.6 \pm 10.2$ & 0.634 \\
GPT (s) & $142.3 \pm 105.2$ & $125.2 \pm 54.0$ & 0.705 \\
Balance (score) & $62.5 \pm 12.4$ & $66.1 \pm 4.0$ & 0.141 \\
\hline
\end{tabular}

$\mathrm{n}=18$

$S P$ silent period, $R M T$ resting motor threshold, EF electric field, $1 O-m a x$ the maximum value of the input-output curve, $1 O-50$ the mid-point of the input-output curve, IO-slope the slope of the input-output curve, TSG total score of gait, BBT Box and Block Test, GPT Grooved Pegboard Test, Pre-LP before lumbar puncture, Post-LP after lumbar puncture

* Significant differences $(p<0.05)$ have been indicated (Wilcoxon signed-rank test)

\section{Discussion}

This is the first iNPH study to characterize the effect of CSF drainage on corticospinal excitability using nTMS. The results showed disturbed inhibition and hyperexcitability in the corticospinal pathways of both upper and lower limbs. As a novel TMS finding in iNPH, we found a significant steepening of the IO-slope in patients who had improved gait performance in the tap test.

The characteristic imaging findings of iNPH may have a substantial impact on TMS-responses due to diseaserelated anatomical alterations affecting the distance from the stimulation coil to the cortex, which alters the effect of TMS according to electromagnetic principles [3, 37]. Thus, previous TMS studies without imaging-based navigation must be interpreted with some caution. One previous study with manually targeted TMS suggested that iNPH patients have decreased RMTs of lower limbs, whereas another study did not find similar results in upper limbs $[10,11]$. In the present study, we paid attention to possible anatomical variations affecting TMS responses and used nTMS with continuous visualization of the stimulation target based on individual MRI anatomy. Nevertheless, in accordance with the previously mentioned non-navigated study, our results showed lowered RMTs in the lower limbs of iNPH patients as compared to the healthy control group [10]. Furthermore, abnormally low RMTs were observed also in the upper limbs of the iNPH patients and the RMTs of upper and 
Table 6 TMS responses and motor parameters before and after lumbar puncture in divided subgroups

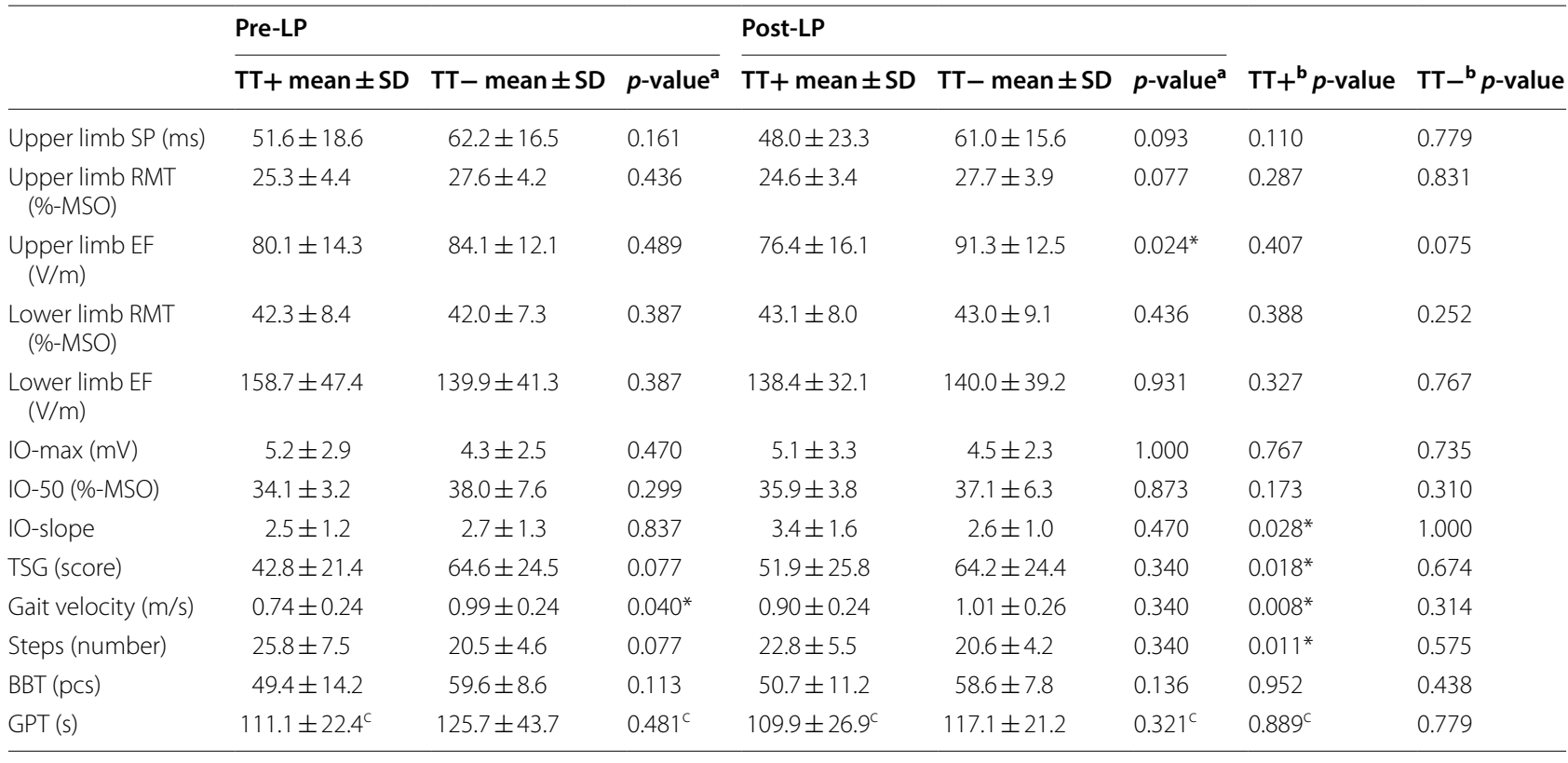

$\mathrm{n}=18$

$S P$ silent period, $R M T$ resting motor threshold, EF electric field, $1 O$-max the maximum value of the input-output curve, $1 O-50$ the mid-point of the input-output curve, IO-slope the slope of the input-output curve, TSG total score of gait, BBT Box and Block Test, GPT Grooved Pegboard Test, Pre-LP before lumbar puncture, Post-LP after lumbar puncture, $T T$ + patients who improved at least 10\% in the TAP test, $T T$ - patients who improved under $10 \%$ in the TAP test

* Indicates significant differences $(p<0.05)$

a Comparison of between-group differences before and after the LP (Independent-Samples Mann-Whitney U test)

b Comparison of within-group differences before and after the LP (Wilcoxon signed-rank test)

c One outlier excluded from statistical analyzes

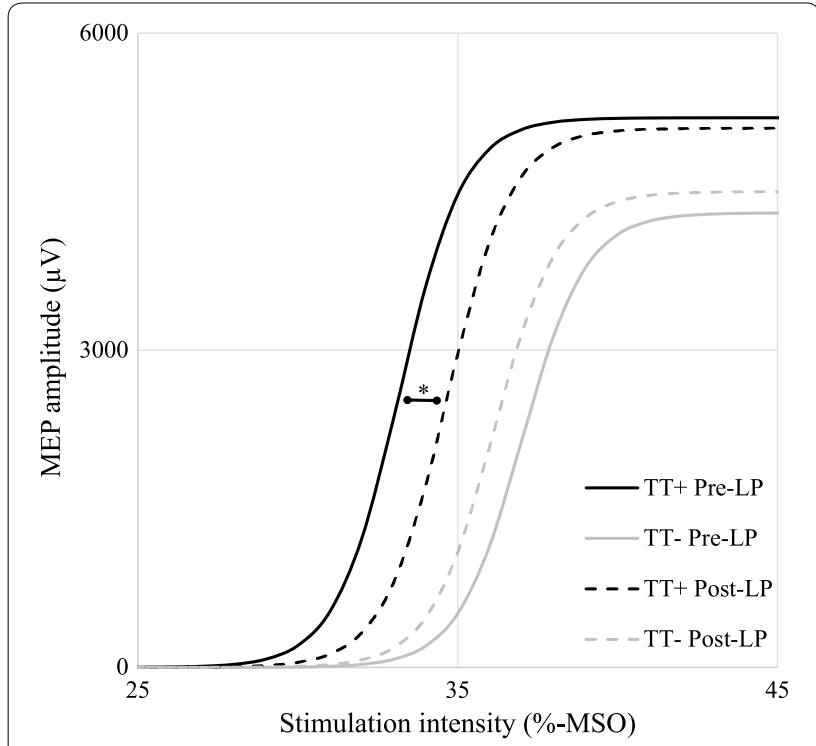

Fig. 3 Input-output curves before and after lumbar puncture with cerebrospinal fluid (CSF) drainage. The slope of the input-output curve steepened in the TT+ subgroup after CSF drainage. *Significant difference, $p=0.028$, Wilcoxon signed-rank test. MEP motor evoked potential, MSO maximum stimulator output. Pre-LP before lumbar puncture, Post- $L P$ after lumbar puncture, $T T+$ patients who improved at least $10 \%$ in the TAP test, TT- patients who improved under $10 \%$ in the TAP test lower limbs correlated with each other. The decreased RMT in the upper limb is a novel finding in iNPH and likely reflects a more generalized dysfunctions of the motor pathways as previously suggested in TMS studies and supports the clinical reports on the involvement of the upper limbs $[8,10]$. In line with the RMT, the EF of the upper limb was also significantly lower in the iNPH group which supports the findings of increased corticospinal excitability, since the EF calculation takes into account stimulator-dependent differences as well as disease-related anatomical alterations affecting the distance from the stimulation coil to the cortex [16, 24]. Previous studies have shown that lower RMTs are associated with a thinning of M1 in healthy subjects which might partly influence our finding, since there are also reports of cortical thinning in iNPH $[47,48]$. Furthermore, in previous studies, lowered RMTs have been hypothesized to derive from increased activity of glutamatergic system [41, 42]. Accordingly, disturbances in glutamatergic system might also underlie the hyperexcitability of corticospinal pathways in iNPH observed in the current study. Interestingly, a recent TMS study indicated that a disturbance in cortical inhibitory cholinergic circuits is related to the 
gait impairment in iNPH which supports the pathophysiological role of the M1 [11].

Our results also showed shortened SPs in iNPH patients which was in line with previous reports $[10,14]$. The duration of the SP has been attributed to cortical inhibitory gamma-aminobutyric acid (GABA) interneurons, mostly $\mathrm{GABA}_{B}[33,43]$. From this perspective, GABAergic mechanisms may also play a role in iNPH resulting in reduced inhibition in corticospinal pathways. The SP duration did not correlate with the RMT, which supports the view that there are multiple contributing mechanisms behind the motor dysfunctions in iNPH. In the current study, corticospinal inhibition measured by SP correlated positively with upper limb gross motor function (BBT). The SP duration was shorter in patients who performed poorer in the BBT which implies that a more severe impairment in upper limb gross motor function is associated with weaker corticospinal inhibition or vice versa. Unexpectedly, the nTMS responses did not correlate with the GPT or gait measurements. TMS induces a direct activation of the corticospinal pathways, and therefore, it could partially bypass the regulatory premotor cortex and the extrapyramidal tracts including the thalamus and the basal ganglia which all are related to the pathophysiology and the motor symptoms of iNPH $[49,50]$. Therefore, the lack of correlation between some TMS responses and the motor parameters may result from a more complex disturbance in the extrapyramidal pathways, which cannot be extensively evaluated by TMS. In agreement with our findings, recent reports have suggested a more widespread motor symptomatology of iNPH with bradykinesia and Parkinson's like symptoms of the upper limbs and these findings challenge the existing practice of focusing on gait as the only routinely assessed motor function $[51,52]$.

In this study, we characterized the immediate effect of LP and CSF drainage on corticospinal excitability. Until now, it has not been addressed whether the treatmentinduced changes of corticospinal excitability in iNPH are a long-term effect of shunt surgery or if they are mediated by the actual decrease in intracranial pressure. Prior studies have shown shunt surgery to restore corticospinal inhibition and to normalize (elevate) RMT in iNPH patients who achieve substantial clinical benefits $[10,14]$. In the present study, patients showed a clinical effect in the TAP test reflected as a significant improvement in TSG, gait velocity and number of steps [28, 29]. However, in the TMS responses no significant changes were observed in SP durations or in RMTs of either upper or lower limb after the LP, whereas an increase in the IOslope was observed in patients who had a positive tap test outcome (TT+ subgroup). In addition, upper limb EFs between $\mathrm{TT}+$ and $\mathrm{TT}-$ subgroups showed a significant difference in the post-LP situation even though no significant change within either subgroup was observed. The IO-slope reflects recruitment of corticospinal output in response to cortical stimulation [44-46]. The steeper IO-slope in patients who improved their gait velocity rapidly after CSF drainage may indicate a higher cortical recovery potential by better preserved synaptic connectivity and plasticity. It might be possible that the previously reported changes in TMS responses after shunt surgery reflect long-term effects of decreased CSF pressure to corticospinal excitability. On the other hand, the IO-slope might be more sensitive in the initial phase after CSF drainage and thus, present an early indicator for the later changes. In diffusion tensor imaging (DTI) studies changes in frontal subcortical white matter tracts, periventricular areas and corpus callosum have been observed after CSF drainage. These DTI findings have been associated with a subsequent clinical improvement and may also underlie the differences in the IO-slopes between the subgroups of the present study [49]. These findings indicate that the background mechanisms of symptom alleviation are more complex than just a mechanic decrease of CSF pressure.

The motor symptoms play a significant role in clinical picture of iNPH but they are only one part of classical symptom triad [1]. The evaluation of cognitive performance and urinary incontinence are beyond the common indications for TMS. The MMSE score as the inclusion criteria is recognized as a limitation of this study. The rationale for requiring a score of 20 or more on the MMSE is based on results of previous research in which the level of attention has been reported to influence TMS parameters [19]. The duration of the TMS protocol was approximately 2 hours during which the patients were required to sit still. Complete muscle relaxation was also required with the exception of the SP evaluation where patients had to activate specific muscle groups on demand. The MMSE score as the inclusion criteria limits the generalization of the results. However, we aimed to recruit a homogeneous study population for this first nTMS study in order to exclude possible confounding factors which might influence TMS parameters. After this pilot study it remains to be verified, whether the results can be generalized in a population which reflects the true clinical variability of iNPH.

\section{Conclusion}

This study showed corticospinal hyperexcitability and impaired corticospinal inhibition in iNPH. The reduced corticospinal inhibition seems to be associated with impaired upper limb motor performance. This finding should be addressed in further studies to evaluate the role of upper limb motor function in the clinical characteristics 
and pathophysiology of iNPH. At baseline, nTMS could not differentiate patients who benefit from CSF drainage but we observed a steepening of the IO-slope in patients who had improved gait performance in the TT. However, further research is required to determine, whether nTMS can offer a biomarker for predicting outcomes of shunt surgery.

\begin{abstract}
Abbreviations
AHB: abductor hallucis brevis; BBT: Box and Block test; CSF: cerebrospinal fluid; DESH: disproportionately enlarged subarachnoid space; DTI: diffusion tensor imaging; EF: electric field; EMG: electromyography; FDI: first dorsal interosseous; GABA: gamma-aminobutyric acid; GPT: Grooved Pegboard Test; iNPH: idiopathic normal pressure hydrocephalus; IO: input-output curve; LP: lumbar puncture; M1: primary motor cortex; MEP: motor evoked potentials; MMSE: Mini-Mental State Examination; MSO: maximum stimulator output; MRI: magnetic resonance image; nTMS: navigated transcranial magnetic stimulation; RMT: resting motor threshold; SP: silent period; TMS: transcranial magnetic stimulation; TSG: total score of gait;TT: Tap test.
\end{abstract}

\section{Acknowledgements}

Not applicable.

\section{Authors' contributions}

JS, LS, PJ, MK, EK, VL and ND drafted and revised the manuscript for content, contributed to the data analysis and interpreted results. The study was designed by VL and ND. JS, LS, MK, EK and ND contributed to the data acquisition. JS performed the statistical analyses. All authors read and approved the final manuscript.

\section{Funding}

JS has received funding from The Finnish Brain Foundation (Helsinki, Finland) The Maire Taponen Foundation (Espoo, Finland), The Cultural Foundation of Northern Savo (Kuopio, Finland) and The Finnish Medical Foundation (Helsinki, Finland).

\section{Availability of data and materials}

The datasets used and analyzed during the current study are available from the corresponding author on reasonable request.

\section{Ethics approval and consent to participate}

The study protocol was approved by the Research Ethics Committee of the Northern Savo Hospital District, 274/2016. All participants gave written informed consent.

\section{Consent for publication}

Not applicable.

\section{Competing interests}

ND has attended a course sponsored by B Braun, distributor of Miethke shunt systems. PJ shared an unrelated patent pending with Nexstim Plc, manufacturer of nTMS systems. LS has received travel bursaries from Nexstim PIc. Other authors report no conflicts of interest.

\section{Author details}

${ }^{1}$ Neurocenter, Neurosurgery, Kuopio University Hospital and University of Eastern Finland, Kuopio, Finland. ${ }^{2}$ Department of Clinical Neurophysiology, Kuopio University Hospital, Kuopio, Finland. ${ }^{3}$ Department of Applied Physics, University of Eastern Finland, Kuopio, Finland. ${ }^{4}$ Department of Clinical Radiology, Kuopio University Hospital, Kuopio, Finland. ${ }^{5}$ Department of Psychiatry, University of Texas Southwestern Medical Center, Dallas, USA. ${ }^{6}$ Unit of Clinical Neuroscience, Neurosurgery, University of Oulu and Medical Research Center Oulu, Oulu University Hospital, Oulu, Finland.

Received: 30 October 2019 Accepted: 3 January 2020

Published online: 17 February 2020

\section{References}

1. Hakim S, Adams RD. The special clinical problem of symptomatic hydrocephalus with normal cerebrospinal fluid pressure: observations on cerebrospinal fluid hydrodynamics. J Neurol Sci. 1965;2(4):307-27.

2. Jaraj D, Rabiei K, Marlow T, Jensen C, Skoog I, Wikkelso C. Prevalence of idiopathic normal-pressure hydrocephalus. Neurology. 2014;82(16):1449-54.

3. Relkin N, Marmarou A, Klinge P, Bergsneider M, Black PM. Diagnosing idiopathic normal-pressure hydrocephalus. Neurosurgery. 2005;57(3):S4-16 (discussion ii-v)

4. Kazui H, Miyajima M, Mori E, Ishikawa M, SINPHONI-2 Investigators. Lumboperitoneal shunt surgery for idiopathic normal pressure hydrocephalus (SINPHONI-2): an open-label randomised trial. Lancet Neurol. 2015;14(6):585-94.

5. Junkkari A, Luikku AJ, Danner N, Jyrkkänen HK, Rauramaa T, Korhonen VE, et al. The Kuopio idiopathic normal pressure hydrocephalus protocol: initial outcome of 175 patients. Fluids Barriers CNS. 2019;16(1):21.

6. Gallagher R, Marquez J, Osmotherly P. Gait and balance measures can identify change from a cerebrospinal fluid Tap test in idiopathic normal pressure hydrocephalus. Arch Phys Med Rehabil. 2018;99:2244-50.

7. Marmarou A, Young HF, Aygok GA, Sawauchi S, Tsuji O, Yamamoto T, et al. Diagnosis and management of idiopathic normal-pressure hydrocephalus: a prospective study in 151 patients. J Neurosurg. 2005;102(6):987-97.

8. Nowak DA, Topka HR. Broadening a classic clinical triad: The hypokinetic motor disorder of normal pressure hydrocephalus also affects the hand. Exp Neurol. 2006;198(1):81-7.

9. Zaaroor M, Bleich N, Chistyakov A, Pratt H, Feinsod M. Motor evoked potentials in the preoperative and postoperative assessment of normal pressure hydrocephalus. J Neurol Neurosurg Psychiatry. 1997;62(5):517-21.

10. Chistyakov AV, Hafner H, Sinai A, Kaplan B, Zaaroor M. Motor cortex disinhibition in normal-pressure hydrocephalus. J Neurosurg. 2012;116(2):453-9.

11. Nardone R, Golaszewski S, Schwenker K, Brigo F, Maccarrone M, Versace $V$, et al. Cholinergic transmission is impaired in patients with idiopathic normal-pressure hydrocephalus: a TMS study. J Neural Transm. 2019;126(8):1073-80.

12. Barker AT, Jalinous R, Freeston IL. Non-invasive magnetic stimulation of human motor cortex. Lancet (London, England). 1985; (8437):1106-7.

13. Ruohonen J, Karhu J. Navigated transcranial magnetic stimulation. Neurophysiol Clin Neurophysiol. 2010;40(1):7-17.

14. Röricht S, Meyer BU, Woiciechowsky C, Lehmann R. Callosal and corticospinal tract function in patients with hydrocephalus: a morphometric and transcranial magnetic stimulation study. J Neurol. 1998;245(5):280-8.

15. Julkunen P, Säisänen L, Danner N, Niskanen E, Hukkanen T, Mervaala E, et al. Comparison of navigated and non-navigated transcranial magnetic stimulation for motor cortex mapping, motor threshold and motor evoked potentials. Neuroimage. 2009;44(3):790-5.

16. Danner N, Julkunen P, Könönen M, Säisänen L, Nurkkala J, Karhu J. Navigated transcranial magnetic stimulation and computed electric field strength reduce stimulator-dependent differences in the motor threshold. J Neurosci Methods. 2008;174(1):116-22.

17. Caipa A, Alomar M, Bashir S. TMS as tool to investigate the effect of pharmacological medications on cortical plasticity. Eur Rev Med Pharmacol Sci. 2018;22(3):844-52.

18. Folstein MF, Folstein SE, McHugh PR. "Mini-mental state":a practical method for grading the cognitive state of patients for the clinician. J Psychiatr Res. 1975;12(3):189-98.

19. De Gennaro L, Marzano C, Veniero D, Moroni F, Fratello F, Curcio G, et al. Neurophysiological correlates of sleepiness: a combined TMS and EEG study. Neuroimage. 2007;36(4):1277-87.

20. Rossi S, Hallett M, Rossini PM, Pascual-Leone A, Safety of TMS Consensus Group TS of TC. Safety, ethical considerations, and application guidelines for the use of transcranial magnetic stimulation in clinical practice and research. Clin Neurophysiol. 2009;120(12):2008-39.

21. Shellock FG, Spinazzi A. MRI safety update 2008: part 2, screening patients for MRI. AJR Am J Roentgenol. 2008;191(4):1140-9.

22. Hashimoto M, Ishikawa M, Mori E, Kuwana N, Study of INPH on neurological improvement (SINPHONI). Diagnosis of idiopathic normal pressure hydrocephalus is supported by MRI-based scheme: a prospective cohort study. Cerebrospinal Fluid Res. 2010;7:18. 
23. Säisänen L, Julkunen P, Niskanen E, Danner N, Hukkanen T, Lohioja T, et al. Motor potentials evoked by navigated transcranial magnetic stimulation in healthy subjects. J Clin Neurophysiol. 2008;25(6):367-72.

24. Danner N, Könönen $M$, Säisänen L, Laitinen R, Mervaala E, Julkunen P. Effect of individual anatomy on resting motor threshold — computed electric field as a measure of cortical excitability. J Neurosci Methods. 2012;203(2):298-304

25. Hellström P, Klinge P, Tans J, Wikkels $\varnothing$ C. A new scale for assessment of severity and outcome in iNPH. Acta Neurol Scand. 2012;126(4):229-37.

26. Morris JC, Heyman A, Mohs RC, Hughes JP, van Belle G, Fillenbaum $\mathrm{G}$, et al. The consortium to establish a registry for Alzheimer's disease (CERAD). Part I. Clinical and neuropsychological assessment of Alzheimer's disease. Neurology. 1989;39(9):1159-65.

27. Mathiowetz V, Volland G, Kashman N, Weber K. Adult norms for the Box and Block Test of manual dexterity. Am J Occup Ther. 1985;39(6):386-91.

28. Wikkelsø C, Andersson $H$, Blomstrand C, Lindqvist $G$. The clinical effect of lumbar puncture in normal pressure hydrocephalus. J Neurol Neurosurg Psychiatry. 1982;45(1):64-9.

29. Marmarou A, Bergsneider M, Klinge P, Relkin N, Black PM. The value of supplemental prognostic tests for the preoperative assessment of idiopathic normal-pressure hydrocephalus. Neurosurgery. 2005;57(3):S1728 (discussion ii-v).

30. Evans WA. An encephalographic ratio for estimating the size of the cerebral ventricles. Am J Dis Child. 1942;64(5):820.

31. Virhammar J, Laurell K, Cesarini KG, Larsson E-M. The callosal angle measured on MRI as a predictor of outcome in idiopathic normal-pressure hydrocephalus. J Neurosurg. 2014;120(1):178-84.

32. Kallioniemi E, Säisänen L, Könönen M, Awiszus F, Julkunen P. On the estimation of silent period thresholds in transcranial magnetic stimulation. Clin Neurophysiol. 2014;125(11):2247-52.

33. Rossini PM, Burke D, Chen R, Cohen LG, Daskalakis Z, Di lorio R, et al. Non-invasive electrical and magnetic stimulation of the brain, spinal cord, roots and peripheral nerves: basic principles and procedures for routine clinical and research application. An updated report from an I.F.C.N. Committee. Clin Neurophysiol. 2015;126(6):1071-107.

34. Kallioniemi E, Savolainen P, Järnefelt G, Koskenkorva P, Karhu J, Julkunen P. Transcranial magnetic stimulation modulation of corticospinal excitability by targeting cortical l-waves with biphasic paired-pulses. Brain Stimul. 2018;11(2):322-6.

35. Awiszus F. TMS and threshold hunting. Suppl Clin Neurophysiol. 2003;56:13-23.

36. Virhammar J, Laurell K, Cesarini KG, Larsson E-M. Increase in callosal angle and decrease in ventricular volume after shunt surgery in patients with idiopathic normal pressure hydrocephalus. J Neurosurg. 2018;130(1):130-5.

37. Julkunen P, Säisänen L, Danner N, Awiszus F, Könönen M. Within-subject effect of coil-to-cortex distance on cortical electric field threshold and motor evoked potentials in transcranial magnetic stimulation. J Neurosci Methods. 2012;206(2):158-64.

38. Fuhr P, Agostino R, Hallett M. Spinal motor neuron excitability during the silent period after cortical stimulation. Electroencephalogr Clin Neurophysiol. 1991;81(4):257-62.
39. Säisänen L, Pirinen E Teitti S, Könönen M, Julkunen P, Määttä S, et al. Factors influencing cortical silent period: optimized stimulus location, intensity and muscle contraction. J Neurosci Methods. 2008;169(1):231-8.

40. Devanne H, Lavoie BA, Capaday C. Input-output properties and gain changes in the human corticospinal pathway. Exp Brain Res. 1997;114(2):329-38.

41. Di Lazzaro V, Oliviero A, Profice P, Pennisi MA, Pilato F, Zito G, et al. Ketamine increases human motor cortex excitability to transcranial magnetic stimulation. J Physiol. 2003:547(2):485-96.

42. Ziemann U, Lönnecker S, Steinhoff BJ, Paulus W. Effects of antiepileptic drugs on motor cortex excitability in humans: a transcranial magnetic stimulation study. Ann Neurol. 1996;40(3):367-78.

43. Groppa S, Oliviero A, Eisen A, Quartarone A, Cohen LG, Mall V, et al. A practical guide to diagnostic transcranial magnetic stimulation: report of an IFCN committee. Clin Neurophysiol. 2012;123(5):858-82.

44. Säisänen L, Julkunen P, Lakka T, Lindi V, Könönen M, Määttä S. Development of corticospinal motor excitability and cortical silent period from mid-childhood to adulthood-a navigated TMS study. Neurophysiol Clin. 2018;48(2):65-75

45. Rosenkranz K, Seibel J, Kacar A, Rothwell J. Sensorimotor deprivation induces interdependent changes in excitability and plasticity of the human hand motor cortex. J Neurosci. 2014;34(21):7375-82.

46. Rosenkranz K, Kacar A, Rothwell JC. Differential modulation of motor cortical plasticity and excitability in early and late phases of human motor learning. J Neurosci. 2007;27(44):12058-66.

47. List J, Kübke JC, Lindenberg R, Külzow N, Kerti L, Witte V, et al. Relationship between excitability, plasticity and thickness of the motor cortex in older adults. Neuroimage. 2013;83:809-16.

48. Kang K, Kwak K, Yoon U, Lee J-M. Lateral ventricle enlargement and cortical thinning in idiopathic normal-pressure hydrocephalus patients. Sci Rep. 2018;8(1):13306

49. Siasios I, Kapsalaki EZ, Fountas KN, Fotiadou A, Dorsch A, Vakharia K, et al. The role of diffusion tensor imaging and fractional anisotropy in the evaluation of patients with idiopathic normal pressure hydrocephalus: a literature review. Neurosurg Focus. 2016;41(3):E12.

50. Townley RA, Botha H, Graff-Radford J, Boeve BF, Petersen RC, Senjem ML, et al. 18 F-FDG PET-CT pattern in idiopathic normal pressure hydrocephalus. Neurolmage Clin. 2018;18:897-902.

51. Krauss JK, Regel JP, Droste DW, Orszagh M, Borremans JJ, Vach W. Movement disorders in adult hydrocephalus. Mov Disord. 1997;12(1):53-60.

52. Molde K, Söderström L, Laurell K. Parkinsonian symptoms in normal pressure hydrocephalus: a population-based study. J Neurol. 2017;264(10):2141-8.

\section{Publisher's Note}

Springer Nature remains neutral with regard to jurisdictional claims in published maps and institutional affiliations.

Ready to submit your research? Choose BMC and benefit from

- fast, convenient online submission

- thorough peer review by experienced researchers in your field

- rapid publication on acceptance

- support for research data, including large and complex data types

- gold Open Access which fosters wider collaboration and increased citations

- maximum visibility for your research: over 100M website views per year

At BMC, research is always in progress.

Learn more biomedcentral.com/submissions 